\title{
Quercetin-3-O-glucoside suppresses pancreatic cancer cell migration induced by tumor-deteriorated growth factors in vitro
}

\author{
JUNGWHOI LEE ${ }^{1}$, JUNGSUL LEE ${ }^{2}$, SEUNG JUN KIM ${ }^{3}$ and JAE HOON KIM ${ }^{1}$ \\ ${ }^{1}$ Faculty of Biotechnology, College of Applied Life Science, SARI, Jeju National University, Jeju-do 63243; \\ ${ }^{2}$ Department of Bio and Brain Engineering, KAIST, Daejeon 34141; ${ }^{3}$ Functional Genomics Research Center, \\ Korea Research Institute of Bioscience and Biotechnology, Daejeon 305806, Republic of Korea
}

Received November 3, 2015; Accepted December 11, 2015

DOI: $10.3892 /$ or.2016.4598

\begin{abstract}
Analysis using Universal exPress Codes (UPCs) with the public microarray database GEO indicates significantly higher mRNA expressions of VEGF-A, bFGF, and bFGFR2 in pancreatic cancers than those in normal pancreatic tissues. Human pancreatic cancer cell line CFPAC-1 and SNU-213 had relatively differential sensitivity to exogenous VEGF-A, bFGF, and TGF- $\beta 1$ in migration property. Treatment of quercetin-3-O-glucoside suppressed the migratory activity induced by TGF- $\beta$ and VEGF-A even at relatively low dosages in CFPAC-1, but not in bFGF-activated SNU-213 cells. However, high dosages of quercetin-3-O-glucoside sufficiently suppressed the migratory activity induced by bFGF in SNU-213 cells. Furthermore, co-treatment with low dose of gemcitabine plus quercetin-3-O-glucoside showed synergistic inhibition effects on the infiltrate activity induced by bFGF in CFPAC-1 and SNU-213 cells. These results collectively suggested that quercetin-3-O glucoside could act as an inhibitor of local metastasis induced by various growth factors in pancreatic cancers and be an effective adjuvant to boost chemotherapeutic efficacy of gemcitabine, currently used in pancreatic cancers.
\end{abstract}

\section{Introduction}

In recent years, an increasing consumption of specific fruit and vegetables containing flavonoids is recognized as the source of health-benefits through their various biochemical properties such as potential anti-carcinogenic and cardioprotective roles (1-3). Ingestion of plant flavonoids is related with decreased risk of various tumors. Several flavonoids are investigated as possible mechanisms of anti-proliferation, antiMMP secretion, anti-migration, anti-invasion, anti-adhesion,

Correspondence to: Dr Jae Hoon Kim, Department of Applied Life Science, Jeju National University, 102 Jejudaehak-ro, Jeju-si, Jeju-do 690-756, Republic of Korea

E-mail:kimjh@jejunu.ac.kr

Key words: quercetin-3-O-glucoside, bFGF, FAK, migration, pancreatic cancer and anti-angiogenic effects (4-8). Some flavonoids are also suggested as potential pro-apoptosis inducers in various cancer cell lines $(9,10)$. Clearly, plants containing various flavonoids are the source of potential therapeutic agents for the benefit of human health.

Pancreatic adenocarcinoma and ductal-adenocarcinoma, the most common, and deadly forms of human pancreatic cancers, are highly invasive and metastatic and associated with poor survival rates $(11,12)$. Migratory activity makes pancreatic cancer therapies difficult with dismal diagnosis and strong chemo-resistance $(13,14)$. Therefore, new agents inhibiting migratory activity of pancreatic adenocarcinoma and ductal-adenocarcinoma are urgently required.

We have previously shown that treatment with quercetin 3-O-glucoside clearly inhibits the migratory activity of pancreatic cancer cells even at relatively low dosages by inhibiting the EGFR signaling pathway (15).

Even though targeting the specific pathway, i.e., EGFR signaling has proven effective in human cancers $(16,17)$, tumors could escape one-direction blockade strategy using alternative signaling pathways (18-20). This competence of tumors allowed us to exploit the additive role of quercetin 3-O-glucoside and its therapeutic potential against various growth factors.

In the present study, we tried to investigate the effects of quercetin-O-3 glucoside on the migratory activity induced by various growth factors in pancreatic cancer cell, and reveal the mechanisms responsible for anti-migratory effects of quercetin-O-3 glucoside. In addition, we also tried to verify its therapeutic potential using combination therapy with gemcitabine treatment. Our results support that quercetin-3-O glucoside can act as an inhibitor of local metastasis induced by different growth factors in pancreatic cancers and could be an effective adjuvant in chemotherapy.

\section{Materials and methods}

Gene expression analysis. Microarray expression profiles were obtained from the Gene Expression Omnibus (GEO) public microarray database. We integrated data-sets independently obtained from several research groups using the absolute normalization method SCAN.UPC (21). The normalization method is dependent on total number of probes; hence, we 
restricted the integration to Affymetrix Human Genome U133 Plus 2.0 Array platform (GPL570) that has a larger number of probes than GPL96 and GPL97. All data were normalized by the default option of SCAN.UPC. In total, 8 data-sets were used i.e., GSE9599, -15471, -16515, -17891, -32676, -39409, -42952 , and -46385 .

Cell culture and reagents. CFPAC-1, pancreatic ductal adenocarcinoma cells were purchased from the American Type Culture Collection (ATCC; Manassa, VA, USA), and SNU-213, pancreatic adenocarcinoma cells were obtained from the Korean Cell Line Bank (KCLB, Seoul, Korea). The cells were cultured in Dulbecco's modified Eagle's medium (DMEM, CFPAC-1) or RPMI-1640 (SNU-213) medium supplemented with $10 \%$ fetal bovine serum (Gibco-BRL, Gaithersburg, MD, USA), and $1 \times 10^{5} \mathrm{U} / 1$ penicillin-100 mg/l streptomycin (Invitrogen, Carlsbad, CA, USA) at $37^{\circ} \mathrm{C}$ in a humidified atmosphere containing $5 \% \mathrm{CO}_{2}$. Antibodies against phospho-FAK (Y397), FAK, phospho-ERK (T202/ Y204), ERK, and GAPDH were obtained from Cell Signaling Technology (Beverly, MA, USA). Suramin sodium, inhibitor of bFGF signaling pathway was purchased from Santa Cruz Biotechnology (Santa Cruz, CA, USA). Quercetin-3-O glucoside and gemcitabine were purchased from Sigma-Aldrich (St. Louis, MO, USA). Recombinant bFGF, VEGF-A, and TGF- $\beta 1$ were purchased from R\&D Systems (Minneapolis, MN, USA).

Migration assay. Cell migration assays were performed as previously described (15). In brief, polycarbonate filters were pre-coated with $10 \mathrm{mg} / \mathrm{l}$ fibronectin (Sigma-Aldrich) in phosphate-buffered saline (PBS), for $30 \mathrm{~min}$ at room temperature. The lower chamber was filled with $500 \mu 1$ of cultured medium containing $10 \%$ serum. After a 24 -h starvation in serum-free medium, cells $\left(5 \times 10^{4}\right.$ cells/well $)$ were suspended in $200 \mu \mathrm{l}$ serum-free medium and loaded into each upper chamber. Cells were then incubated for $4 \mathrm{~h}$ at $37^{\circ} \mathrm{C}$. Filters were fixed with $4 \%$ paraformaldehyde and stained with $1 \%$ crystal violet solution. The absorbance of the eluted dye was measured at $560 \mathrm{~nm}$ in an ELISA reader (Bio-Rad, Richmond, CA, USA).

Western blotting. Western blotting was performed as previously described to evaluate phosphorylation of various molecules (22). The bands were measured by densitometry using Image J software (National Institutes of Health, Bethesda, MD, USA).

Statistical analysis. Data are presented as means \pm standard deviation (SD). The level of significance for comparisons between two independent samples was determined using Student's t-tests. Groups were compared using a one-way analysis of variance (ANOVA) with Tukey's post-hoc test for significant main effects (SPSS 12.0K for Windows; SPSS Inc., Chicago, IL, USA).

\section{Results}

Expression levels of various growth factors in human pancreatic cancers. To select manifested-signaling pathways in human pancreatic cancer cells (HPCCs), we performed mRNA analysis using Universal Express Codes (UPCs) with the public microarray database GEO. Pancreatic cancers expressed significantly more bFGF and VEGF-A mRNAs, as compared with normal pancreatic tissues. Specifically, pancreatic cancers expressed particularly higher levels of bFGF, as compared with the normal pancreatic samples. In contrast, there was no significant difference in TGF- $\beta 1$ expression levels between pancreatic cancers and normal pancreatic samples (Fig. 1A). The receptors of VEGF-A, bFGF, and TGF- $\beta 1$ expression profiles were also examined using the public microarray database GEO. As shown Fig. 1B, FGFR2 expression level was elevated in pancreatic cancer samples. However, FLT-1, TGF- $\beta \mathrm{R} 1$, and TGF- $\beta \mathrm{R} 2$ expression levels were similar in both pancreatic cancer samples and normal pancreatic tissues. Interestingly, the expression level of FLK-1 was significantly lower in pancreatic cancer samples, as compared with normal pancreatic tissues. These results clearly showed that pancreatic cancers has different expression patterns of growth factors and its receptors including unique expression patterns of bFGF signaling-related molecules, bFGF and FGFR2.

Treatment of quercetin-3-O glucoside decreased the migratory activity induced by different growth factors in human pancreatic cancer cells. Human pancreatic cancer cells (SNU-213 and CFPAC-1) were exogenously treated with bFGF, TGF- $\beta 1$, and VEGF-A under the absence or presence of relatively low dosages of quercetin-3-O glucoside to investigate the anti-migratory effects of quercetin-3-O glucoside against exogenous activations. In SNU-213 cells, TGF- $\beta 1$ or VEGF-A-induced migration was inhibited by quercetin 3-O-glucoside even at low dosages (50 and $100 \mathrm{nM}$ ); the same treatment caused a weak effect in bFGF-treated SNU-213 cells (Fig. 2A). In contrast, there was a significant inhibitory effect in bFGF-induced migration in CFPAC-1 cells (Fig. 2B). Interestingly, exogenous TGF- $\beta 1$ and VEGF-A treatment did not induce migration activity in CFPAC-1 cells.

We next investigated the involvement of bFGF signaling pathway using the pharmaceutical bFGF blocker, suramine sodium, on SNU-213 cell migration. As shown Fig. 3, bFGF treatment-induced cell migration was dose-dependently inhibited by suramine pre-treatment. Moreover, co-treatment of suramine and quercetin-3-O glucoside significantly decreased the bFGF-induced migration (suramine; $100 \mathrm{nM}$, quercetin3-O glucoside; $100 \mathrm{nM}$ ).

Different modes of quercetin-3-O glucoside treatment for anti-migratory effect in human pancreatic cancer cells. bFGF-treated SNU-213 cells were assayed for migration activity in the presence of various concentration of quercetin 3-O-glucoside $(0,100,500,1,000,10,000$, and 100,000 nM) to investigate the strategy of the effective blockade of bFGF-induced migration in SNU-213 cells. Treatment of quercetin-3-O-glucoside significantly decreased bFGF-induced migration of SNU-213 cells up to $35 \%$, as compared with that in exogenously bEGF-treated control cells in a dose-dependent manner (Fig. 4).

We further evaluated whether treatment of quercetin 3-O-glucoside can have synergistic anti-migratory effect when combined with gemcitabine, a currently used reagent in pancreatic cancer therapy. At the highest concentration tested $(1,000 \mathrm{nM})$, gemcitabine alone significantly reduced 

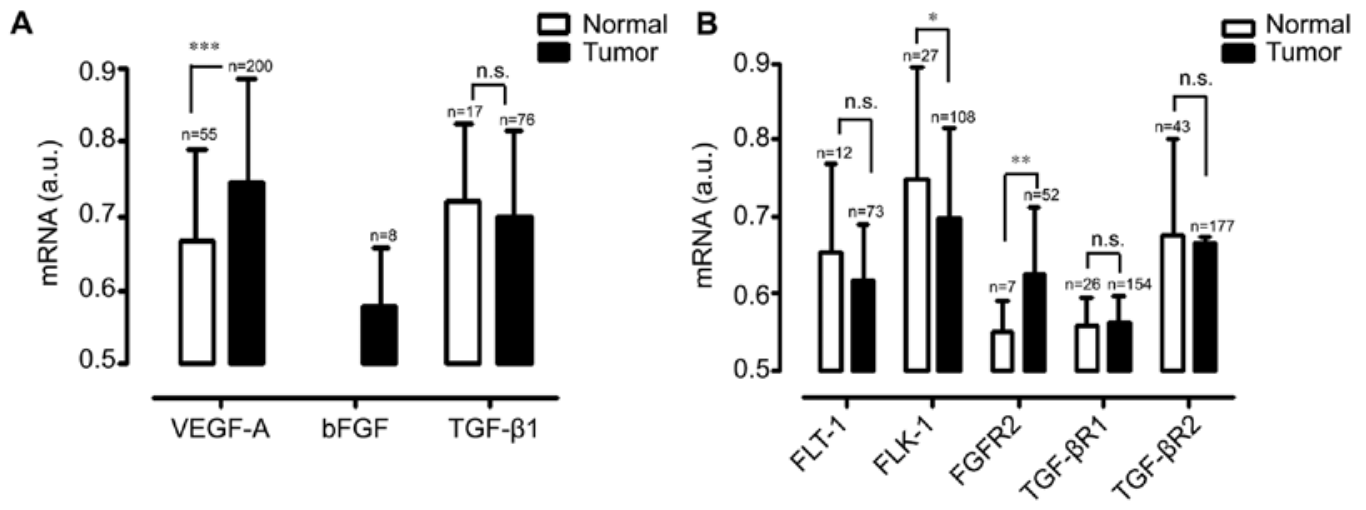

Figure 1. Various growth factors and the receptor expression using GEO databases. (A) The transcriptional levels of VEGF-A, bFGF, and TGF- $\beta 1$ in pancreatic cancers and normal pancreatic samples were analyzed by the Gene Expression Omnibus (GEO) databases. (B) The transcriptional levels of FLT-1, FLK-1, FGFR2, TGF- $\beta$ R1, and TGF- $\beta$ R2 in pancreatic cancers and normal pancreatic samples were analyzed by the Gene Expression Omnibus (GEO) database.

A

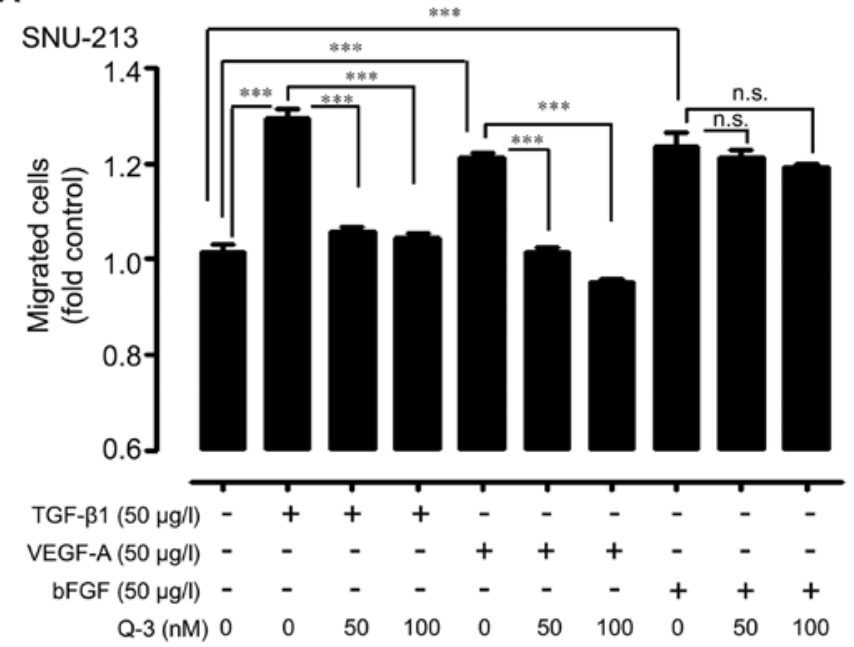

B

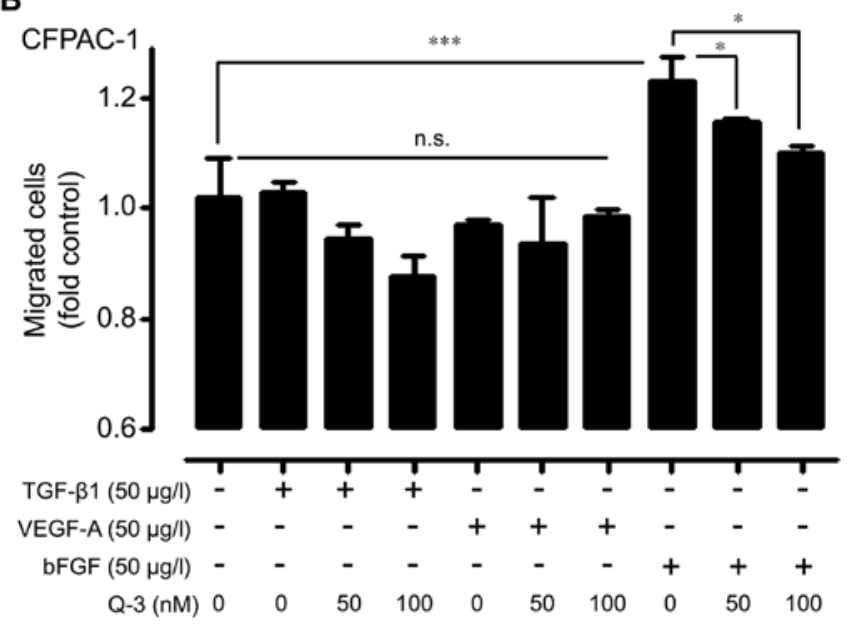

Figure 2. The sensitivity of quercetin-3-O glucoside treatment in growth factor-induced migration in human pancreatic cancer cells. (A and B) SNU-213 cells and CFPAC-1 cells pre-incubated with different doses of quercetin-3-O-glucoside were treated with $50 \mu \mathrm{g} / 1$ of exogenous TGF- $\beta 1$, VEGF-A, or bFGF. Migration activity was evaluated using the Transwell-migration assay ( $n=3$; Tukey's post hoc test was applied to the significant group effects in ANOVA, $\mathrm{P}<0.0001$; asterisks indicate a significant difference, as compared to $0 \%$ inhibition, ${ }^{*} \mathrm{P}<0.05,{ }^{* * *} \mathrm{P}<0.001$, n.s., non-significant values statistically).

A

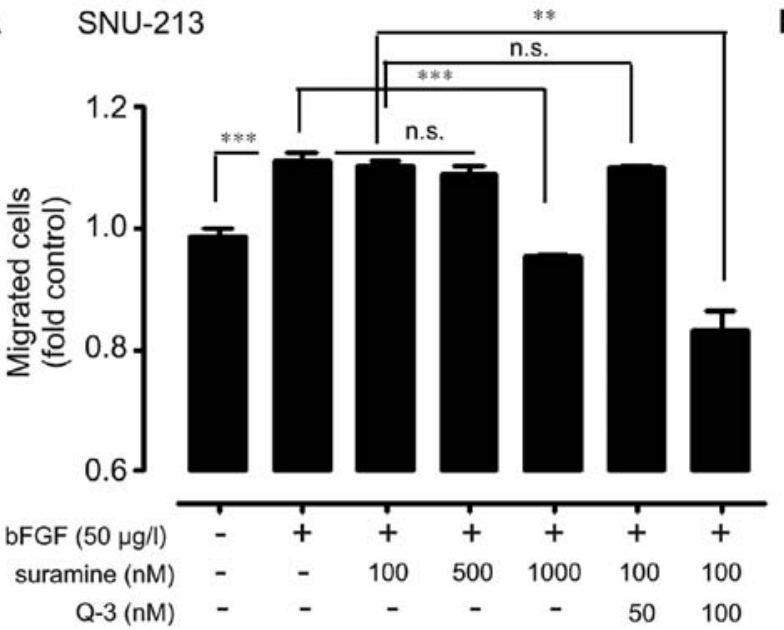

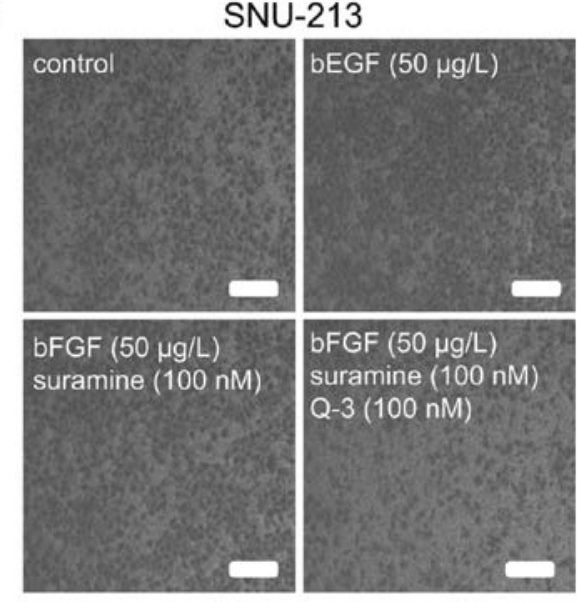

Figure 3. Combined effects of quercetin-3-O glucoside and inhibitors of bFGF in human pancreatic cancer cells. (A) SNU-213 cells were incubated with varying doses of suramine and different doses of quercetin-3-O glucoside (50 and $100 \mathrm{nM})$. Migration activity was evaluated using the Transwell-migration assay ( $\mathrm{n}=3$; Tukey's post hoc test was applied to the significant group effects in ANOVA, $\mathrm{P}<0.0001$; asterisks indicate a significant difference, as compared to $0 \%$ inhibition, ${ }^{*} \mathrm{P}<0.05,{ }^{* *} \mathrm{P}<0.01,{ }^{* * *} \mathrm{P}<0.001$ ). (B) Representative image of a Transwell migration assay co-treated with quercetin-3-O-glucoside, suramine, and bFGF (scale bar, $50 \mu \mathrm{m}$ ). 
A

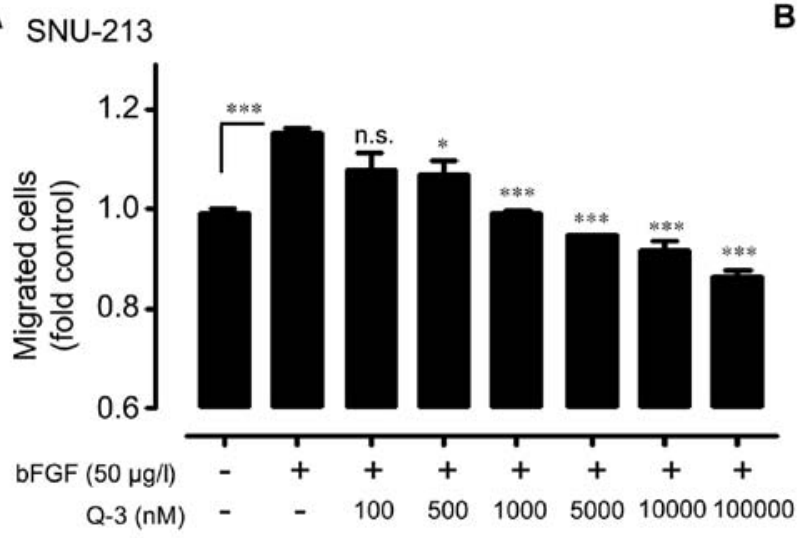

B

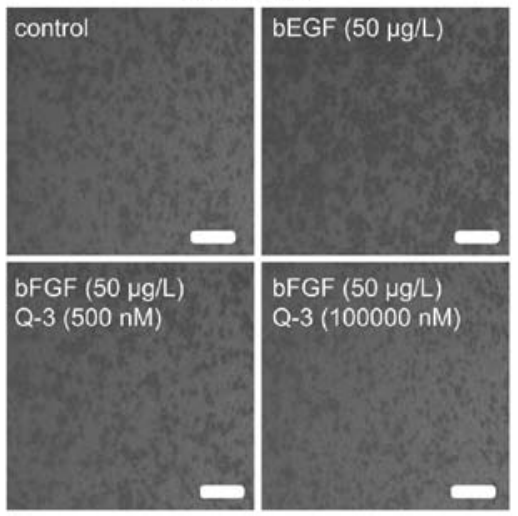

Figure 4. Dose-dependent anti-migratory effect of quercetin-3-O glucoside. (A) SNU-213 human pancreatic cancer cells were pre-treated with different doses of quercetin-3-O-glucoside and then incubated with $50 \mu \mathrm{g} / \mathrm{l}$ bFGF. Migration was evaluated using the Transwell migration assay (n=3; Tukey's post-hoc test was applied to detect the significant group effects as determined by analysis of variance, $\mathrm{P}<0.0001 ;{ }^{*} \mathrm{P}<0.05,{ }^{* * *} \mathrm{P}<0.001$ vs. $0 \%$ inhibition, $\mathrm{n} . \mathrm{s}$., non-significant values statistically). (B) Representative image of the Transwell migration assay treated with 500 or 100,000 nM of quercetin-3-O-glucoside and EGF (scale bar, $50 \mu \mathrm{m}$ ).

A

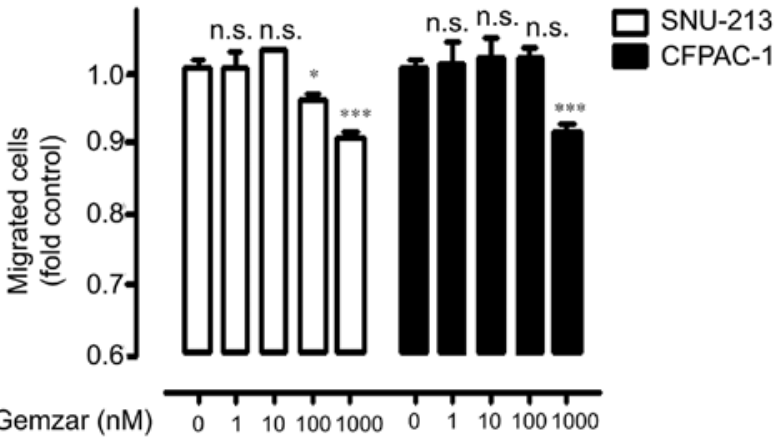

B

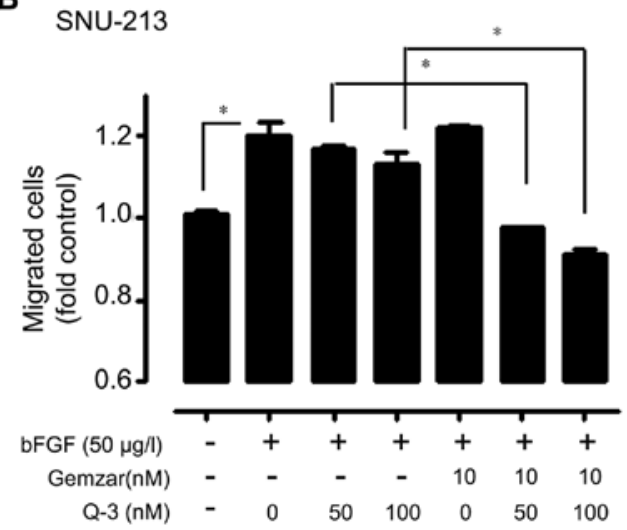

C

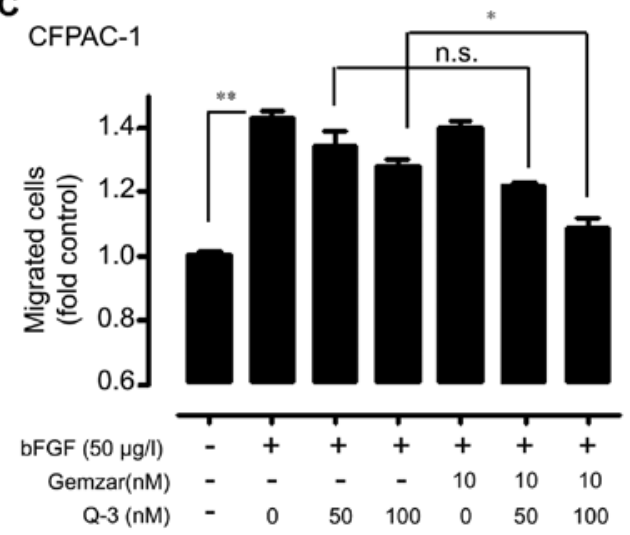

Figure 5. Combined effects of quercetin-3-O glucoside and gemcitabine in in human pancreatic cancer cells. (A) SNU-213 and CFPAC-1 cells were incubated with varying doses of gemcitabine for $6 \mathrm{~h}$. Migration activity was evaluated using the Transwell-migration assay ( $\mathrm{n}=3$; Tukey's post hoc test was applied to significant group effects in ANOVA, $\mathrm{P}<0.0001$; asterisks indicate a significant difference compared to $0 \%$ inhibition, ${ }^{*} \mathrm{P}<0.05$, $\left.{ }^{* *} \mathrm{P}<0.01\right)$. $(\mathrm{B}) \mathrm{Exogenous} b \mathrm{bGF}$ $(50 \mu \mathrm{g} / \mathrm{l})$ was treated in different doses of quercetin-3-O-glucoside alone or quercetin-3-O-glucoside and gemcitabine combined pre-incubated SNU-213 cells. Migration activity was evaluated using the Transwell-migration assay ( $\mathrm{n}=3$; Tukey's post hoc test was applied to the significant group effects in ANOVA, $\mathrm{P}<0.0001$; asterisks indicate a significant difference compared to $0 \%$ inhibition, $\left.{ }^{*} \mathrm{P}<0.05,{ }^{* *} \mathrm{P}<0.01\right)$. (C) Exogenous bFGF (50 $\left.\mu \mathrm{g} / \mathrm{l}\right)$ was treated in different doses of quercetin-3-O-glucoside alone or quercetin-3-O-glucoside and gemcitabine combined pre-incubated CFPAC-1 cells. Migration activity was evaluated using the Transwell-migration assay ( $\mathrm{n}=3$; Tukey's post hoc test was applied to significant group effects in ANOVA, $\mathrm{P}<0.0001$; asterisks indicate a significant difference compared to $0 \%$ inhibition, $\left.{ }^{*} \mathrm{P}<0.05,{ }^{* *} \mathrm{P}<0.01\right)$.

the cell migrations in SNU-213 and CFPAC-1 cells, while the low doses of gemcitabine had little effect (Fig. 5A). We chose the $10 \mathrm{nM}$ concentration of gemcitabine for a combinatory-strategy. SNU-213 and CFPAC-1 cells were incubated in the absence or presence of gemcitabine $(10 \mathrm{nM})$ with different doses of quercetin 3-O-glucoside $(0,50$, and $100 \mathrm{nM})$ and bFGF $(50 \mu \mathrm{g} / \mathrm{l})$. As shown in Fig. 5B, the SNU-213 cell migration was significantly reduced up to $22 \%$ by $100 \mathrm{nM}$ quercetin 3-O-glucoside treatment. Under the same conditions, CFPAC-1 cell migration was also reduced 
A

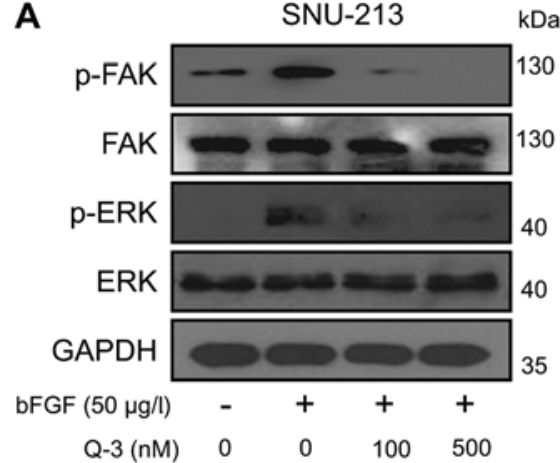

B

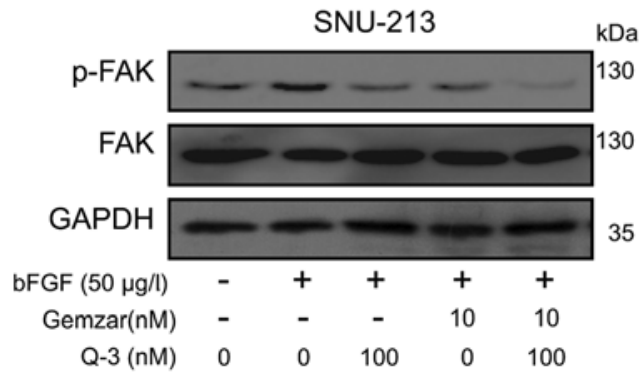

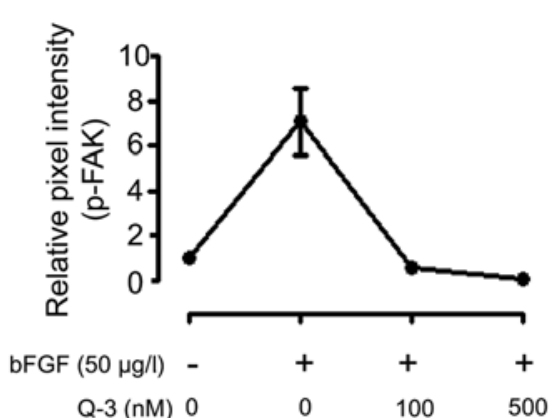
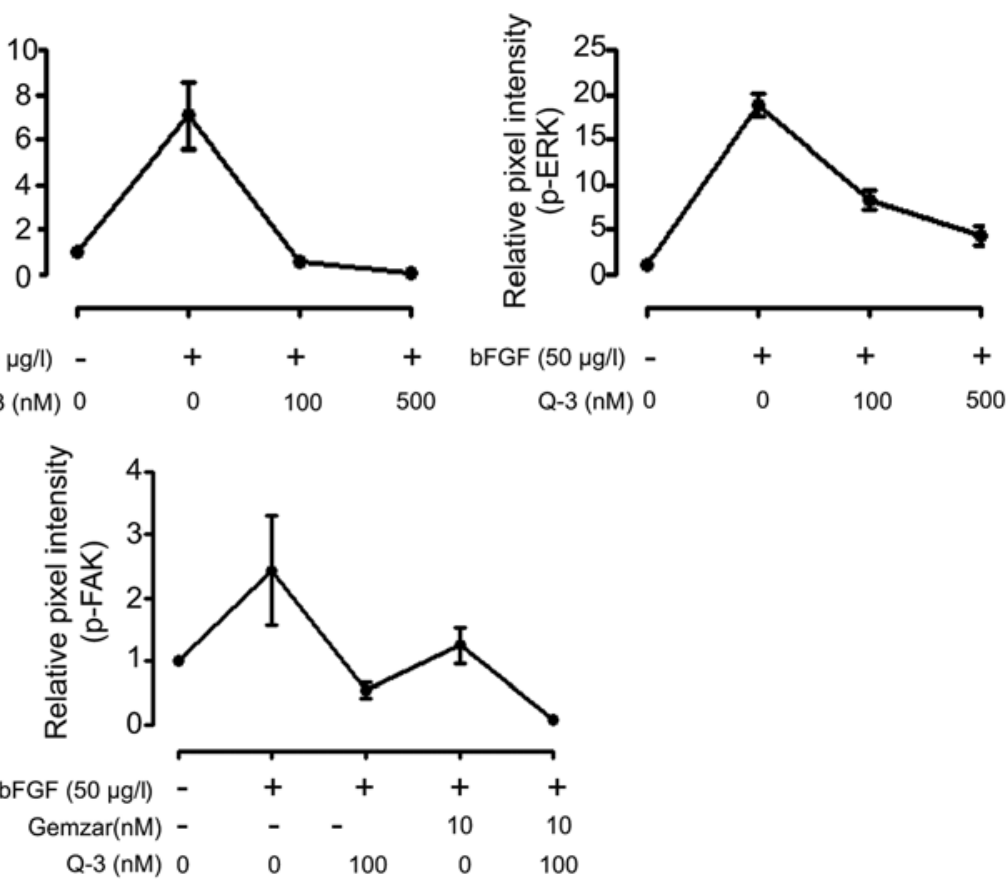

Figure 6. Intracellular signaling in response to treatment of quercetin-3-O glucoside and co-treatment with gemcitabine. (A) Left, SNU-213 cells were pretreated with different doses of quercetin-3-O-glucoside for $1 \mathrm{~h}$ followed by treatment with exogenous bFGF (50 $\mu \mathrm{g} / \mathrm{l})$ for $30 \mathrm{~min}$ and the cell lysates were subjected to western blot analysis using antibodies specific to phosphor FAK (Y397), FAK, phosphor-ERK1/2 (T202/Y204), ERK1/2, and GAPDH Right, relative pixel intensity for p-FAK and p-ERK was measured using p-FAK/GAPDH or p-ERK2/GAPDH (B) Left, SNU-213 cells pre-incubated with quercetin-3-O-glucoside alone or quercetin-3-O-glucoside and gemcitabine combined, were treated with $50 \mu \mathrm{g} / \mathrm{l}$ of exogenous bFGF for $30 \mathrm{~min}$ and the cell lysates were subjected to western blot analysis using antibodies specific to phosphor FAK (Y397), FAK, and GAPDH. Right, relative pixel intensity for p-FAK was measured using $\mathrm{p}-\mathrm{FAK} / \mathrm{GAPDH}$.

up to $15 \%$ (Fig. 5C). These results collectively demonstrated that high dosage treatment of quercetin3-O glucoside or low dosage co-treatment of quercetin3-O glucoside with gemcitabine show effective anti-migratory effects in bFGF activated human pancreatic cancer cells.

Treatment of quercetin 3-O-glucoside inhibits bFGF-induced migratory activity through the blockade of ERK1/2 signaling pathway in human pancreatic cancer cells. The migration inhibitory effects induced by quercetin 3-O-glucoside in bFGF activated human pancreatic cancer cells were evaluated in the study of bFGF signaling pathway with effective dose of quercetin 3-O-glucoside. Exogenous bFGF treatment activated the phosphorylation levels of FAK and ERK1/2; while pretreatments of quercetin 3-O-glucoside significantly decreased phosphorylation level of FAK and ERK1/2 (Fig. 6A). In addition, co-treatments of quercetin3-O glucoside and gemcitabine significantly inhibited FAK phosphorylation, as compared with quercetin3-O glucoside or gemcitabine single treatment in bFGF activated human pancreatic cancer cells (Fig. 6B). These results suggested that combination therapy of quercetin3-O glucoside and gemcitabine induces synergistic anti-migratory effects in bFGF activated human pancreatic cancer cells through the blockade of FAK and ERK1/2 signaling pathway.

\section{Discussion}

In the present study, we demonstrated that pancreatic cancers have relatively high mRNA expression patterns of various growth factors and its receptor such as VEGF-A, bFGF, and FGFR2, as compared to normal pancreatic samples using the public microarray database GEO. Furthermore, quercetin-3-O-glucoside can effectively inhibit human pancreatic cancer cells migration induced by various growth factors such as VEGF-A, bFGF, and TGF- $\beta$. We further showed that co-treatment of quercetin-3-O-glucoside with gemcitabine had a synergic effect to suppress the migratory activity in human pancreatic cancer cells through the inhibition of FAK and ERK1/2 signaling pathway. These results suggested that the naturally-occurring antioxidant, quercetin 3-O-glucoside, might be a cancer cell migratory inhibitor and could be used in adjuvant therapy.

Multiple phenotypes are caused by reciprocal interactions of various growth factors and its receptors in most cancers including pancreatic cancers. We have previously reported that quercetin 3-O-glucoside effectively inhibits the EGF-induced migratory activity of human pancreatic cancer cells by inhibiting the EGFR signaling pathway (15). Even though targeting the EGFR signaling pathway has been proven effective in human cancers $(16,17)$, some tumors could escape the one way blockade strategy using alternative signaling pathways (18-20). In the present study, human pancreatic cancer cells thus had a differential dependency to various growth factor activations such as VEGF, bFGF, and TGF- $\beta$ and treatment of quercetin-3-O-glucoside also had a differential migratory-inhibition effects in SNU-213 and CFPAC-1 cells (23). Especially, exogenous bFGF treatment resulted in different responses to quercetin-3-O-glucoside 
between SNU-213 and CFPAC-1 cells. Previous studies have shown that enhanced bFGF expression is correlated with pancreatic cancer stages in vitro and in vivo, resistance to chemotherapy, and selection of cancer targeting candidates (24-28). In agreement with these studies, our results suggested that elevated bFGF signaling leads to an increased chemoresistance in SNU-213 cells. As proof-of-concept, mRNA analysis correctly identified different responses to various growth factors. Therefore, to overcome the resistance to quercetin-3-O-glucoside treatment, we demonstrated the strategy for blockade of bFGF-induced migration using relatively high-dose treatments within the safe range of quercetin-3-O-glucoside and co-treatment of quercetin 3-O-glucoside and suramine with reducing the potential risk of side-effect in SNU-213 cells. Treatment with low dosages of suramine seems to sensitize the responses to relatively low dosages of quercetin 3-O-glucoside. Further detailed experiments are needed to confirm these findings.

Subsequently, quercetin-3-O-glucoside showed a synergic anti-migratory effect with relatively low dosage of gemcitabine. To date, gemcitabine is still regarded as the standard treatment for pancreatic cancer patients despite its controversial effects. Combinational trials with gemcitabin and cytotoxic reagents, i.e., 5-FU, cisplatin, oxaloplatin, and capectabine (29-32) and biological reagents, i.e., erlotinib, cetuximab, and bevacizumab (33-35) have been reported. Some of those treatments had effective outcomes; however, none of the combination trials were significantly more effective, as compared with gemcitabin alone.

Despite the significant improvement in overall survival rates in other cancers during the last few decades, the prognosis of pancreatic cancers is unfortunately at a standstill $(36,37)$, with an almost $100 \%$ mortality in pancreatic cancer patients. The dismal prognosis in pancreatic cancer is mainly due to its high metastatic potential, the difficulty of diagnosis, and its high chemo-resistance (38). Clearly, most pancreatic cancer patients are entirely disadvantaged from the most commonly used gemcitabine through the chemo-resistance and metastatic behavior (39).

According to the present study, quercetin-3-O-glucoside can act as the inhibitor of local metastasis induced by various growth factors in pancreatic cancers and is the effective co-treatment with gemcitabine for intractable pancreatic cancer treatment, despite its phytochemical origin in intractable pancreatic cancer cells.

\section{Acknowledgements}

This research was supported by the Basic Science Research Program through the National Research Foundation of Korea (NRF) funded by the Ministry of Education (2009-0094059).

\section{References}

1. Formica JV and Regelson W: Review of the biology of quercetin and related bioflavonoids. Food Chem Toxicol 33: 1061-1080, 1995.

2. Surh YJ: Cancer chemoprevention with dietary phytochemicals. Nat Rev Cancer 3: 768-780, 2003.

3. No authors listed: Diet, nutrition and the prevention of chronic diseases. World Health Organ Tech Rep Ser 916: i-viii, 1-149, 2003.
4. Rodriguez J, Yáñez J, Vicente V, Alcaraz M, Benavente-García O, Castillo J, Lorente J and Lozano JA: Effects of several flavonoids on the growth of B16F10 and SK-MEL-1 melanoma cell lines: Relationship between structure and activity. Melanoma Res 12: 99-107, 2002.

5. Vijayababu MR, Arunkumar A, Kanagaraj P, Venkataraman P, Krishnamoorthy G and Arunakaran J: Quercetin downregulates matrix metalloproteinases 2 and 9 proteins expression in prostate cancer cells (PC-3). Mol Cell Biochem 287: 109-116, 2006.

6. Lee WJ, Wu LF, Chen WK, Wang CJ and Tseng TH: Inhibitory effect of luteolin on hepatocyte growth factor/scatter factor-induced HepG2 cell invasion involving both MAPK/ERKs and PI3K-Akt pathways. Chem Biol Interact 160: 123-133, 2006.

7. Kamaraj S, Anandakumar P, Jagan S, Ramakrishnan G and Devaki T: Modulatory effect of hesperidin on benzo(a)pyrene induced experimental lung carcinogenesis with reference to COX-2, MMP-2 and MMP-9. Eur J Pharmacol 649: 320-327, 2010.

8. Balakrishnan A and Menon VP: Effect of hesperidin on matrix metalloproteinases and antioxidant status during nicotine-induced toxicity. Toxicology 238: 90-98, 2007.

9. Sivagami G, Vinothkumar R, Bernini R, Preethy CP, Riyasdeen A, Akbarsha MA, Menon VP and Nalini N: Role of hesperetin (a natural flavonoid) and its analogue on apoptosis in HT-29 human colon adenocarcinoma cell line - a comparative study. Food Chem Toxicol 50: 660-671, 2012.

10. Ghorbani A, Nazari M, Jeddi-Tehrani M and Zand H: The citrus flavonoid hesperidin induces p53 and inhibits NF- $\kappa$ B activation in order to trigger apoptosis in NALM-6 cells: Involvement of PPAR $\gamma$-dependent mechanism. Eur J Nutr 51: 39-46, 2012.

11. Smith K: Pancreatic cancer: FASCINating insights into the metastatic nature of pancreatic cancer. Nat Rev Gastroenterol Hepatol 11: 139, 2014.

12. Hermann PC, Huber SL, Herrler T, Aicher A, Ellwart JW, Guba M, Bruns CJ and Heeschen C: Distinct populations of cancer stem cells determine tumor growth and metastatic activity in human pancreatic cancer. Cell Stem Cell 1: 313-323, 2007.

13. Bardeesy $\mathrm{N}$ and DePinho RA: Pancreatic cancer biology and genetics. Nat Rev Cancer 2: 897-909, 2002.

14. Cano CE, Motoo Y and Iovanna JL: Epithelial-to-mesenchymal transition in pancreatic adenocarcinoma. Sci World J 10: 19471957, 2010.

15. Lee J, Han SI, Yun JH and Kim JH: Quercetin 3-O-glucoside suppresses epidermal growth factor-induced migration by inhibiting EGFR signaling in pancreatic cancer cells. Tumour Biol 36: 9385-9393, 2015.

16. Harari PM: Epidermal growth factor receptor inhibition strategies in oncology. Endocr Relat Cancer 11: 689-708, 2004.

17. Vokes EE and Chu E: Anti-EGFR therapies: Clinical experience in colorectal, lung, and head and neck cancers. Oncology (Williston Park) 20 (Suppl 2): 15-25, 2006.

18. Wajed SA, Laird PW and DeMeester TR: DNA methylation: An alternative pathway to cancer. Ann Surg 234: 10-20, 2001.

19. Tabernero J: The role of VEGF and EGFR inhibition: Implications for combining anti-VEGF and anti-EGFR agents. Mol Cancer Res 5: 203-220, 2007.

20. Wang S, Pashtan I, Tsutsumi S, Xu W and Neckers L: Cancer cells harboring MET gene amplification activate alternative signaling pathways to escape MET inhibition but remain sensitive to Hsp90 inhibitors. Cell Cycle 8: 2050-2056, 2009.

21. Piccolo SR, Withers MR, Francis OE, Bild AH and Johnson WE: Multiplatform single-sample estimates of transcriptional activation. Proc Natl Acad Sci USA 110: 17778-17783, 2013.

22. Lee J, Ku T, Yu H, Chong K, Ryu SW, Choi K and Choi C: Blockade of VEGF-A suppresses tumor growth via inhibition of autocrine signaling through FAK and AKT. Cancer Lett 318: 221-225, 2012.

23. Lee J, Lee J, Yu H, Choi K and Choi C: Differential dependency of human cancer cells on vascular endothelial growth factormediated autocrine growth and survival. Cancer Lett 309: 145-150, 2011.

24. Yamanaka Y, Friess H, Buchler M, Beger HG, Uchida E, Onda M, Kobrin MS and Korc M: Overexpression of acidic and basic fibroblast growth factors in human pancreatic cancer correlates with advanced tumor stage. Cancer Res 53: 5289-5296, 1993.

25. Nomura S, Yoshitomi H, Takano S, Shida T, Kobayashi S, Ohtsuka M, Kimura F, Shimizu H, Yoshidome H, Kato A, et al: FGF10/FGFR2 signal induces cell migration and invasion in pancreatic cancer. Br J Cancer 99: 305-313, 2008. 
26. Lieu C, Heymach J, Overman M, Tran H and Kopetz S: Beyond VEGF: Inhibition of the fibroblast growth factor pathway and antiangiogenesis. Clin Cancer Res 17: 6130-6139, 2011.

27. Wagner M, Lopez ME, Cahn M and Korc M: Suppression of fibroblast growth factor receptor signaling inhibits pancreatic cancer growth in vitro and in vivo. Gastroenterology 114: 798-807, 1998.

28. Zhang H, Hylander BL, LeVea C, Repasky EA, Straubinger RM, Adjei AA and Ma WW: Enhanced FGFR signalling predisposes pancreatic cancer to the effect of a potent FGFR inhibitor in preclinical models. Br J Cancer 110: 320-329, 2014.

29. Berlin JD, Catalano P, Thomas JP, Kugler JW, Haller DG and Benson AB III: Phase III study of gemcitabine in combination with fluorouracil versus gemcitabine alone in patients with advanced pancreatic carcinoma: Eastern Cooperative Oncology Group Trial E2297. J Clin Oncol 20: 3270-3275, 2002.

30. Heinemann V, Quietzsch D, Gieseler F, Gonnermann M, Schönekäs H, Rost A, Neuhaus H, Haag C, Clemens M, Heinrich B, et al: Randomized phase III trial of gemcitabine plus cisplatin compared with gemcitabine alone in advanced pancreatic cancer. J Clin Oncol 24: 3946-3952, 2006.

31. Louvet C, Labianca R, Hammel P, Lledo G, Zampino MG, André T, Zaniboni A, Ducreux M, Aitini E, Taïeb J, et al, GERCOR; GISCAD: Gemcitabine in combination with oxaliplatin compared with gemcitabine alone in locally advanced or metastatic pancreatic cancer: Results of a GERCOR and GISCAD phase III trial. J Clin Oncol 23: 3509-3516, 2005.

32. Herrmann R, Bodoky G, Ruhstaller T, Glimelius B, Bajetta E, Schüller J, Saletti P, Bauer J, Figer A, Pestalozzi B, et al; Swiss Group for Clinical Cancer Research; Central European Cooperative Oncology Group: Gemcitabine plus capecitabine compared with gemcitabine alone in advanced pancreatic cancer: A randomized, multicenter, phase III trial of the Swiss Group for Clinical Cancer Research and the Central European Cooperative Oncology Group. J Clin Oncol 25: 2212-2217, 2007.
33. Moore MJ, Goldstein D, Hamm J, Figer A, Hecht JR, Gallinger S, Au HJ, Murawa P, Walde D, Wolff RA, et al; National Cancer Institute of Canada Clinical Trials Group: Erlotinib plus gemcitabine compared with gemcitabine alone in patients with advanced pancreatic cancer: A phase III trial of the National Cancer Institute of Canada Clinical Trials Group. J Clin Oncol 25: 1960-1966, 2007.

34. Cascinu S, Verdecchia L, Valeri N, Berardi R and Scartozzi M: New target therapies in advanced pancreatic cancer. Ann Oncol 17 (Suppl 5): v148-v152, 2006.

35. Kindler HL, Friberg G, Singh DA, Locker G, Nattam S, Kozloff M, Taber DA, Karrison T, Dachman A, Stadler WM, et al: Phase II trial of bevacizumab plus gemcitabine in patients with advanced pancreatic cancer. J Clin Oncol 23: 8033-8040, 2005.

36. Li D, Xie K, Wolff R and Abbruzzese JL: Pancreatic cancer. Lancet 363: 1049-1057, 2004.

37. Koay EJ, Truty MJ, Cristini V, Thomas RM, Chen R, Chatterjee D, Kang Y, Bhosale PR, Tamm EP, Crane CH, et al: Transport properties of pancreatic cancer describe gemcitabine delivery and response. J Clin Invest 124: 1525-1536, 2014.

38. Stathis A and Moore MJ: Advanced pancreatic carcinoma: Current treatment and future challenges. Nat Rev Clin Oncol 7: 163-172, 2010.

39. Long J, Zhang Y, Yu X, Yang J, LeBrun DG, Chen C, Yao Q and Li M: Overcoming drug resistance in pancreatic cancer. Expert Opin Ther Targets 15: 817-828, 2011. 